\title{
CONTRA LA INSTRUMENTALIZACIÓN DE LA EDUCACIÓN SUPERIOR EN MÉXICO
}

Marveya Villalobos Torres

\section{RESUMEN}

El objetivo de este escrito es mostrar que la calidad ${ }^{1}$ constituye el eje vertebrador del "Programa Nacional de Educación 2001-2002" (PNE). Los cinco gobiernos anteriores se habían centrado en la cobertura, atendiendo a la demanda y no a la necesidad, lo que ha representado una falacia en nuestro sistema educativo.

Asimismo, queremos llevar a cabo una lectura política de la Pedagogía presentada en el PNE (2001-2006) en el subprograma de Educación Superior, el cual se expresa en el lineamiento a seguir durante este sexenio - que concluye en el 2006 - y que como afirmaba, desde el título de sus obras, el doctor Ernesto Meneses Morales son alternativamente "Tendencias de la educación en México (...)".

Nuestro argumento se centra en no instrumentalizar la Educación Superior, sustentándola en un modelo que calificaríamos de "mercado", diferente al de su orientación social².

${ }^{1}$ El PNE 2001-2006 lo incluye refiriéndose a un aspecto filosófico. Ninguno de los programas anteriores, al menos los cinco últimos, incluye un apartado de cuestionamiento filosófico — sustento de la calidad educativa-; nos referimos a los gobiernos de Ernesto Zedillo (1994-2000), Carlos Salinas de Gortari (1988-1994), Miguel de la Madrid (1982-1988), José López Portillo (1976-1982) y Luis Echeverría Álvarez (1970-1976).

${ }^{2}$ La Universidad es la conciencia crítica de la sociedad. Además de sus funciones sustantivas —docencia, investigación científica, humanística y tecnológica; estudios tecnológicos; y extensión, preservación y difusión de la cultura, según la misión antropológica de cada una-, tiene una misión más elevada: la búsqueda de la verdad y de la formación integral de personas humanas. 
En una primera aproximación, señalaremos que este análisis subraya que el PNE es la expresión de la política educativa actual en un sistema que pugna por la transición hacia la democracia. En una segunda aproximación, se presenta el subprograma de Educación Superior, suscrito en treinta y cinco páginas de las doscientos sesenta y nueve que posee en total. Como tercera, los comentarios críticos desde las tendencias de los organismos pedagógicos internacionales y de la situación real de México, sus problemas y retos en el ámbito educativo. Y en una cuarta aproximación, concluiremos con propuestas concretas que eviten la instrumentalización de la Educación Superior en México.

\section{SUBPROGRAMA DE LA EDUCACIÓN SUPERIOR CO- MO EXPRESIÓN DE LA POLÍTICA EDUCATIVA FOXISTA}

La política educativa se agrupa en directrices que señalan los gobiernos para el sector educativo en el marco de su política general, partidista o nacionalista ${ }^{3}$. Esta política ofrece los criterios y la orientación que deben inspirar a los elementos constitutivos de todo sistema educativo: finalidades, metas y objetivos; organización administrativa; estructura pedagógica; contenidos educativos; métodos, técnicas y procedimientos; así como el perfil de los autores del proceso enseñanza-aprendizaje.

La política educativa tiene dos referentes: la planificación — programación de la acción con vistas a lograr un objetivo ${ }^{4}$ y la legislación que la regula, en este caso la Ley General de Educación. En el primer referente se encuentra ubicado el "Programa Nacional de Educación 2001-2006".

"La política educativa presenta la actividad del poder público dirigida a solucionar los problemas sociales de la educación, por lo que en el fondo se convierte en un conjunto de medidas de la política general, cuyo objetivo específico es, en todas sus dimensiones, el políticon.

${ }^{3}$ Cfr. Santillana, Diccionario de Ciencias de la Educación, p.1118.

${ }^{4}$ Cfr., RAYNAL, F., Pédagogie: dictionnaire des concepts clés., p.286.

${ }^{5}$ COLOM., et.al., Introducción a la Política de la Educación., p.20. 
Todos los gobiernos presentan, a partir del Plan Nacional de Desarrollo, los programas sectoriales. De esta forma: "La política educativa se nos presenta con un discurso educativo de ámbito muy generalizado; todos los Estados regulan directrices y legislan para el sector educativo, dentro del marco de su política general ${ }^{6}$.

La política educativa no es una disciplina asentada en un constructo teórico, o en un planteamiento filosófico exclusivo del mundo de las ideas o de los sistemas; la política educativa existe en la realidad; forma parte de lo que Habermas denominó «mundo de la vida" y viene dotada de realidad por la voluntad de grupos sociales concretos que, en cada momento histórico, tienen la necesidad y la pertinente responsabilidad de asumir el logro de unos objetivos, y que representa la suma o el complejo de múltiples dimensiones: corrientes de pensamiento, valores humanos y sociales, objetivos económicos, necesidades culturales y sociales.

En este contexto, y después de nueve meses de asumir el poder, el presidente Vicente Fox presentó, en el Palacio de Bellas Artes el viernes 28 de septiembre de 2001, el «Programa Nacional de Educación 2001-2006" y afirmó: "Nada define mejor el sentido de una obra de gobierno que su política educativa ${ }^{7}$.

También en esta presentación, se comprometió a elevar a 8\% del PIB destinado a la educación ${ }^{8}$.

En una situación de transición democrática, como la que México vive, es donde mayor interés adquiere la política educativa, los niveles de libertad y la necesaria transparencia de la propia gestión política. Esto hace que grupos políticos, académicos y sociales se interesen principalmente por la cuestión de los grandes principios formativos y por la aplicación de los diferentes programas; por ello, en el democrático, más que en ningún otro contexto político, se requiere arbitrar, al tiempo, mecanismos de participación y mecanismos de evaluación y control?.

\footnotetext{
${ }^{6}$ Ibidem., p. 21.

${ }^{7}$ Fox, Vicente., Discurso de Presentación de la PNE 2001-2006 el viernes 28 de septiembre de 2001.

${ }^{8}$ Actualmente se otorga el 4.67\%. Será muy difícil lograrlo, porque el $70 \%$ del PIB está destinado a pago de intereses de la deuda interna y externa del país. ${ }^{9}$ Cfr. COLOM, J., et.al., op.cit., p.27.
} 
El programa educativo del sexenio fue sintetizado por el propio presidente de México: "Equidad, sí, pero con calidad; calidad, sí, pero con evaluación externa; evaluación, sí, pero con rendición de cuentas para su verificación; rendición de cuentas, sí, pero con la participación corresponsable de los ciudadanos y la comunidad educativa ${ }^{10}$.

No hay duda de que la educación, desde múltiples perspectivas - y por supuesto también desde la política-, se ha ido convirtiendo en un "Centro de Interés" y de debate social. La política educativa es parte imprescindible de la formación pedagógica actual; lo pedagógico no sólo tiene capacidad de ofertar una lectura política, sino que su razón de ser — su propia realidad en la escuela, así como las perspectivas de futuro que inmanentemente se encuentran en la actuación educativa - sólo puede ser comprendida en toda su identidad desde el análisis político.

La historia educativa está implicada en las políticas educativas de cada momento histórico; la realidad de nuestras universidades, de nuestro sistema educativo —e incluso muchos aspectos a simple vista puramente pedagógicos como el currículum, el uso de las distintas tecnologías educativas, las estrategias didácticas, etcétera- obedecen indudablemente al protagonismo de las decisiones políticas.

La política educativa del gobierno actual (2001-2006) contempla el ayer, el presente y el futuro de la educación; de hecho, engloba y explicita toda la fenomenología pedagógica concurrente, hasta tal punto que puede ser considerada como una forma de entender y analizar el fenómeno educativo en todas sus dimensiones.

Asimismo, la Educación Comparada ofrece una forma nueva de hacer Pedagogía, la cual es una perspectiva de análisis que ofrece la estructura conceptual de la propia Pedagogía. La metodología de la Educación Comparada es una perspectiva

${ }^{10}$ FOX, V., op.cit., viernes 28 septiembre de 2001. 
capaz de englobar múltiples aspectos pedagógicos; ello aporta una lectura política de la Pedagogía, así como una comprensión desde la política de la educación ${ }^{11}$.

Hasta aquí, hicimos referencia a la cultura propia de la política educativa, que nos ofrece el contexto de la realidad política de la educación: desde la planificación, en donde se inscribe el PNE: 2001-2002, y desde la legislación u ordenamiento del marco jurídico.

\section{PROGRAMA NACIONAL DE EDUCACIÓN 2001-2006. SUBPROGRAMA EDUCACIÓN SUPERIOR}

Este apartado intenta describir lo comprendido en el subprograma de Educación Superior. Presentamos las bases para el programa 2001-2006 del sector educativo, realizado por la coordinación del Área Educativa del equipo de transición el Presidente electo ${ }^{12}$ cuyo objetivo número 13 es:

Ampliar la cobertura del sistema de educación superior y convertirlo en la fuerza impulsora del crecimiento económico y el desarrollo social del país.

Este objetivo incluye siete líneas estratégicas:

1. Fomentar la mejora continua e integral de la calidad del sistema de educación superior.

2. Impulsar un nuevo enfoque educativo centrado en el aprendizaje y caracterizado por la innovación, flexibilidad, movilidad, atención y formación integral de alumnos y alumnas.

3. Promover la operación de un sistema abierto e integrado que desarrolle nuevas formas de vinculación y extensión con su entorno, y con el sistema de ciencia y tecnología, en beneficio de una mayor pertinencia y calidad.

4. Promover la expansión y diversificación del sistema mediante la creación de nuevas instituciones públicas con los perfiles académicos requeridos.

${ }^{11}$ Cfr. COLOM, J., op.cit., p.30.

${ }^{12}$ Lunes 27, noviembre de 2000. Periódico Reforma. Realizado en cinco meses del 2 de julio a 27 de noviembre de 2000 . 
5. Ampliar y fortalecer los programas de formación y actualización de profesores y profesoras.

6. Fomentar que las instituciones y sus programas académicos sean evaluados y, en su caso, acreditados por organismos externos especializados, reconocidos por el Estado.

7. Asegurar que las instituciones públicas cuenten con los recursos públicos requeridos para funcionar con calidad y promover que amplíen su base financiera.

Lo anterior es la fuente directa que inspira lo presentado como subprograma de Educación Superior, el cual inicia con una introducción. El discurso de este exordio sustenta a la educación como un "medio" para el "hacer" y no se refiere al fin ni al ser.

Enfatiza las funciones sustantivas de las Instituciones de Educación Superior (IES) y las caracteriza en el contexto de la necesidad de la cobertura, calidad y equidad. Asimismo, considera la necesidad de lograr la eficiencia terminal con altas tasas de titulación o graduación. Aspira a pasar de un sistema "cerrado" a uno "abierto", "flexible", "innovador" y "dinámico", en el proceso de enseñanza-aprendizaje; para ello propone crear una "sistema nacional de becas", con la finalidad de lograr la calidad, centrándose en la superación académica de los profesores y en la actualización de los contenidos con un enfoque educativo centrado en el aprendizaje. De igual forma, hace referencia a la reestructuración de la planeación de educación superior. Antes de continuar con un diagnóstico del sistema de educación superior, se enuncia el orden metodológico del subprograma: políticas, objetivos estratégicos, líneas de acción y metas ${ }^{13}$.

El diagnóstico se centra sólo en el orden infraestructural, número de instituciones, programas, proyectos financiados por el Programa para el Mejoramiento de Profesores (PROMEP), equipamiento, etcétera.

${ }^{13}$ Sobre la base de un análisis comparativo, sólo se desarrollan: Las Bases para el Programa 2001-2006 del sector educativo, que ya están referidos con antelación en este escrito. 
Surge un cuestionamiento: ¿desarrollo, sin financiamiento y sin valoración cualitativa? Enseguida se enuncian los principales problemas y retos de la educación superior centrados en equidad y cobertura, calidad y gestión.

El siguiente rubro hace referencia a la visión de la educación superior a 2025, presentado en cinco párrafos, sin ninguna referencia a las tendencias que "recomiendan" los Organismos Pedagógicos Internacionales ${ }^{14}$.

Por último, el PNE presenta tres objetivos estratégicos - reiterando los indicadores de cobertura con equidad, calidad y gestión - desarrollando en cada uno las políticas, los objetivos particulares, las líneas de acción y las metas.

\section{COMENTARIOS CRÍTICOS DEL SUBPROGRAMA DE EDUCACIÓN SUPERIOR}

La característica de PNE es de equilibrio entre continuidad e innovación. Se fundamenta en lo propuesto por el equipo de transición basándose en el objetivo y las siete líneas estratégicas.

Reforma y modernización es la característica de PNE, sin alusión a la Revolución Educativa ni a la pretensión de reinventar.

La propia Secretaría de Educación Pública (SEP) define al PNE como "Propuesta viva" y perfectible ${ }^{15}$ y reitera el deseo de fomentar el diálogo sistemático entre investigadores y tomadores de decisión ${ }^{16}$ (base sobre la cual se sustenta nuestro comentario,

\footnotetext{
${ }^{14}$ Profundizar en: UNESCO., La educación superior en el siglo XXI., Visión y Acción. Conferencia Mundial sobre Educación Superior, octubre 1998.

UNESCO, Foro Mundial sobre la Educación. Dapor, Senegal, Abril de 2000. UNESCO., El Derecho a la Educación. 2000.

vid., UNESCO., Les clés du XXIe Siecle., préface de Koichiro Matsura, directeur genéral D`UNESCO., Sevil, París.,2000.,515.

Es necesario hacer patente que la visión al 2025 está dada por la UNESCO basándose en una serie de recomendaciones y que la OCDE ofrece la ayuda económica para llevarlo a la acción.

${ }^{15}$ SEP., Programa Nacional de Educación., p.240.

${ }^{16}$ Ibidem., p.101.
} 
que intenta buscar y encontrar salidas viables para el logro del perfeccionamiento de la educación superior en México). En primer lugar, lo más preocupante de instrumentalizar la educación para el "hacer" o el "tener" — como mecanismo de movilidad social - es que «la educación está hecha de convicciones arraigadas en la inteligencia y en la voluntad (...) al ritmo de nuestras decisiones en el ámbito del ser (...)" ${ }^{17}$. Así, es precisamente lo permanente, la esencia, lo que le ofrece asidero auténtico a la educación. "Lo mismo pasa con instituciones y sociedades, hay elementos cambiantes que son posibles gracias a una base de elementos permanentes, constantes ${ }^{18}$.

Fundamentarse en lo "permanente" de la educación es la base para lograr la tan ansiada calidad; hoy, la gran mayoría de las Instituciones de Educación Superior (IES) viven y sufren el síndrome del "cambio".

Es paradójico que las metas propuestas para la educación superior en cobertura, calidad y gestión, estén marcadas entre el 2001 (que además ya terminó) y el 2006, y la visión que recomienda la UNESCO es para el 2025; el PNE no presenta visión para el 2007. Inferencia: ¿vivimos la tendencia del sexenio, una vez más?

Sin embargo, las universidades e instituciones de educación superior viven un proceso de transformación silenciosa. Se trata de cambios profundos a diferentes niveles, que abarcan funciones sustantivas y organizativas, que de forma desigual se han desarrollado desde afuera (vía las políticas gubernamentales) o desde dentro de las instituciones, pero que ya definen un conjunto de orientaciones y procesos de funcionamiento.

Durante los últimos veinte años ha predominado la mutación en las IES y, con ello, los cambios de los paradigmas del conocimiento de aquello que había prevalecido en por lo

${ }^{17}$ GONZÁLES-SIMANCAS, J.L., Lo permanente y lo cambiante en la educación., p.51. ${ }^{18}$ PICOS, A.E., "¿Es posible transformar lo humano?"., en ISTMO., Año 44., No. 259., Marzo-abril 2002, 28-31 p. 
menos un siglo ${ }^{19}$. El Banco Mundial hace referencia a que el $64 \%$ de la riqueza de todo el mundo proviene del capital humano ${ }^{20}$.

Todo ello da cuenta de su actual importancia económica y de la adquisición de un conjunto de habilidades, destrezas, capacidades, competencias y calificaciones que son aprehendidas en las instituciones educativas; esto evidencia que hoy se vive el cambio de los paradigmas del conocimiento. En nuestros días, la academia debe propiciar el generar un conocimiento capaz de interpretar, usar, concebir y producir; la clave es volverlo pertinente para transferirlo, es decir centrarse en lo permanente, en el "para qué"; ser consciente de cómo se está aprehendiendo.

Actualmente, las IES tienen una tarea clara: redefinir su oferta, su demanda y las condiciones básicas de funcionamiento y operación. La política de modernización conservadora del gobierno foxista exige estar en la lógica del mercado y de la competitividad. Desde la campaña de Fox, la educación superior fue señalada como fundamental para la creación de "capital humano" y como eje central de la competitividad del país con el mundo. La condición es adoptar la calidad, tal y como se presenta en las empresas productivas y de servicio; ella es la clave para orientar los cambios en las instituciones. El compromiso es: "Contagiar la pasión por la calidad”. Las IES públicas carecen de un espíritu empresarial como para operar con un esquema ideal de mercado y competitividad.

\footnotetext{
${ }^{19}$ La mutación fundamental a la que nos referimos es que no es todo ni cualquier tipo de conocimiento el que tiene hoy utilidad en el mercado, sino sólo cierto tipo de conocimiento, el que posee "significado diferente" que ofrece la habilidad, la competencia y la capacidad social para generarlo; producir estas nuevas formas de conocimiento se ha constituido en el componente fundamental en el proceso de cambio que viven las IES. Éste es el eje articulador en la actualización que exige el diseño curricular de los programas de Educación Superior.

${ }^{20} \mathrm{NEEF}$, Dale., The Knowledge Economy., p.2.
} 
Hoy son muchos los actores involucrados en el futuro de la Universidad, la "Sociología de la Universidad se preocupa sistemáticamente por el futuro de la Universidad ${ }^{21}$, o con mayor precisión por los futuros de la Universidad ${ }^{22}$.

La ANUIES, inquieta por el futuro de las universidades, se propuso, desde 1998, crear una nueva visión del sistema educativo mexicano desde un enfoque prospectivo (en el contexto que las IES se encontraban impulsando importantes cambios en el nivel institucional y hacía falta plantear un conjunto de líneas estratégicas para su desarrollo), cuyo resultado es el documento "ANUIES. La Educación Superior en el Siglo XXI. Líneas Estratégicas de Desarrollo. Una Propuesta de la ANUIES,23.

Se recomienda un estudio acucioso del capítulo tercero, caracterizado por lo deseable pero no constructivo del enfoque prospectivo de la educación superior.

LA ANUIES construye una visión para las IES al año 2020, basándose en ocho postulados: 1) calidad e innovación; 2) congruencia con su naturaleza académica; 3) pertinencia en relación con las necesidades del país; 4) humanismo; 5) compromiso con la construcción de una sociedad mejor; 6) autonomía responsable; 7) estructura de gobierno y; 8) operaciones ejemplares.

El documento de ANUIES presenta un diagnóstico, basado en otros indicadores diferentes al PNE: a) demográfico, en donde augura para el 2020 la creación de muchas universidades; b) crecimiento urbano y, por ende, creación de desorden; c) el factor económico lo adjetiva, caracterizándolo por la incertidumbre; d) laboral-empleo, enfatiza que se desarrollará el factor de servicios, más que el industrial y el sector primario, lo cual exigirá capacidad de autoaprendizaje para toda la vida, trabajo en equipo, capacidad de compromiso y sensibilidad

${ }^{21}$ Nos ha parecido sumamente interesante incluir el análisis propuesto por la ANUIES en educación superior.

${ }^{22}$ TORRES, Juan Ángel., La Universidad Virtual., p. 24

${ }^{23}$ México, 2000, 266 p. 
social, así como aptitud para resolver problemas; e) desarrollo nacional y regional.

Una vez más, ANUIES realiza un diagnóstico reduccionista y limitativo a lo cuántico, con escasez de concreción en cuanto a pertinencia social, currículo, organización académica, gestión, legislación, calidad e innovación, y lo más grave: eminentemente descriptivo en el rubro de la investigación y la cooperación internacional. Destaca en su visión crítica del proceso enseñanza-aprendizaje por su tradicionalidad, obsolencia, rigidez y falta de innovación y creatividad. Es insuficiente en eficiencia y eficacia de la gestión institucional ${ }^{24}$.

En el orden cuantitativo se presentan cuatro escenarios para el año 2020, con un corte en el 2006.

La ANUIES, así como el PNE propuesto por la SEP, omiten citar los dos postulados propuestos por la UNESCO para reorientar el sistema de Educación Superior sobre: la sociedad del conocimiento y la adopción del paradigma de la educación permanente y para toda la vida ${ }^{25}$. Asimismo, en las

${ }^{24}$ Las dimensiones reconocidas y aceptadas para realizar el análisis de un sistema educativo son:

1) Eficiencia externa o pertinencia o relevancia. Mide el grado en que el sistema educativo provee los conocimientos, aprendizajes, habilidades y destrezas requeridas en el mercado laboral (formal y no formal), así como los valores y actitudes que reclama una sociedad.

2) Eficiencia interna: a) flujos educativos y; b) asignación y consumo de todo tipo de recursos (dinero, personas, cosas y tecnología).

3) Efectividad o calidad. Mide el grado de adquisición, por parte de los estudiantes, de los conocimientos, valores, actitudes y competencias que fijan oficialmente los planes y programas de estudio de un país.

4) Equidad. Mide la diferencia en las oportunidades de acceso, permanencia y egreso del sistema educativo en todos sus niveles y modalidades, así como en la calidad del servicio educativo ofrecido a diferentes grupos poblacionales.

5) Capacidad de gestión. Mide las reformas administrativas. Se manifiesta por la capacidad de fijar políticas, monitorear la ejecución, evaluar resultados e impactos y la asignación de recursos.

Estas dimensiones son utilizadas como evidencias empíricas en el marco internacional; son más evidentes en países desarrollados que en países en vías de desarrollo como México.

Para profundizar, revisar PRAWDA, J. y FLORES, G., México Educativo Revisitado., México., 2001., 317 p.

${ }^{25}$ UNESCO., Les clés du XXIe Siecle., p. 282. 
recomendaciones financieras realizadas por el Banco Mundial (BM), el Banco Interamericano de Desarrollo (BID) y por la Organización para la Cooperación del Desarrollo Económico (OCDE).

Todo el documento de ANUIES postula una transformación, pero sustentada en un cambio de carácter tendencial y formal. Sólo innovación, reforma, pero no transformación.

En su propuesta prospectiva hay una ausencia de análisis sobre escenarios múltiples para el futuro de las IES; sólo existe uno deseable y, además, presentado como el único bueno y por lo tanto utópico.

La propuesta de la ANUIES hacia un sistema abierto se centra en la innovación curricular y pedagógica de forma generalizada, con mayor crecimiento de la matrícula.

Por ende no hay modelo, y califica de tipo ideal de corte weberiano ${ }^{26}$ entre lo abierto y lo cerrado.

El paso hacia un sistema abierto, se reduce a una abstracción que se pretende consolidar con el claustro de profesores y de los investigadores.

En cuanto al paradigma del aprendizaje ${ }^{27}$ (también postulado de la UNESCO) se reduce a un discurso con aseveraciones más formales que sustanciales.

Por otro lado, es excesivo en cuanto a la evaluación externa del documento; no se comprende el significado de impulsar el autoestudio en las instituciones. Ello tuvo impacto en la década de los setenta y ochenta; hoy se han constituido en cumplimientos formales.

ANUIES señalaba, en la década de los noventa, que el financiamiento para la educación superior sería del 1.5\% del PIB. En el PNE se propone un incremento anual hasta alcanzar el 1\% para el 2006 (lo planteado desde el proceso de transición era llegar al 2\%).

\footnotetext{
${ }^{26}$ ANUIES., op.cit., p.187.

${ }^{27}$ vid., UNESCO, Les clés du XXIe Siecle., p.276.

${ }^{28}$ vid., ULLOA, Manuel., El Financiamiento de la Educación Superior Pública en México. (Mim 2001).
} 
Hasta aquí el contexto de ANUIES que, al estudiarlo, sirve como referente para el análisis los tres objetivos estratégicos del PNE 2001-2006 en lo correspondiente al tipo educativo superior $^{29}$.

\section{AMPLIACIÓN DE LA COBERTURA CON EQUIDAD}

En primer lugar, es necesario señalar que la desigualdad social se refleja en la estructura del sistema educativo mexicano.

Es fundamental reconocer que el debate internacional en educación superior tiene como tendencia un viraje radical y una transformación de fondo en las IES ${ }^{30}$; en base a un «modelo de mercado" que tienda a la diversificación en las modalidades, niveles y tipos, que responda a las demandas de la sociedad y de la economía nacional, lo cual está manifiesto en las MISIONES de las IES ${ }^{31}$.

En cuanto a la cobertura, el equipo de transición del sector educativo propuso: pasar de una tasa del 18\% del grupo de edad correspondiente a educación superior al 28\%, aunque no se explicita de qué sectores sociales. Esto significa que se deberá llegar a dos millones ochocientos mil estudiantes en 2006. El problema, es que no hay políticas que amplíen los niveles actuales de la educación en poblaciones marginales indígenas, además de que no existe financiamiento.

Por lo tanto, por ahora es más o menos estable. Estudiar el PNE en cuanto a las metas propuestas, hacia los dos años de gobierno foxista, se visualizan diluidas.

\footnotetext{
${ }^{29}$ La certeza de la complejidad de la realidad dentro de las universidades mexicanas no puede resumirse a la cobertura, calidad y gestión.

${ }^{30}$ UNESCO., Conferencia Mundial sobre Educación Superior., París., Octubre., 1998.

${ }^{31}$ Hasta la fecha no se puede hacer referencia en el contexto de la diversificación a: "Universidades de investigación" o "Universidades de Posgrado". Bastaría realizar un estudio de educación comparada con respecto de Estados Unidos.
} 


\section{EDUCACIÓN SUPERIOR DE BUENA CALIDAD}

El $\mathrm{PNE}^{32}$ propone que un sistema de educación superior de buena calidad es aquél orientado a satisfacer las necesidades del desarrollo social, científico, tecnológico, económico, cultural y humano del país; es promotor de innovaciones y se encuentra abierto al cambio en entornos institucionales caracterizados por la argumentación racional rigurosa, responsabilidad, tolerancia, creatividad y libertad; cuenta con una cobertura suficiente, una oferta amplia y diversificada que atiende a la demanda educativa con equidad, con solidez académica y eficiencia en la organización y utilización de sus recursos".

Asimismo, el equipo de transición del sector educativo concretó la educación en un enfoque flexible y eficiente, basado en el aprendizaje, así como en atender al desarrollo humano integral, a la formación en valores y a una disciplina intelectual.

Surge el cuestionamiento: ¿cuál es la orientación pedagógica y curricular a la que se hace referencia?

Una de las metas propuestas para lograr los postulados anteriores es duplicar el número de profesores para alcanzar una relación de 12.5 estudiantes por profesor, de los cuales el 34\% de tiempo completo de éstos — casi el 50\%— tendrán Posgrado. Para lograrlo, habrá 10,000 becas para realizar Posgrados. ¿Cómo será la calidad de los estudios que se ofrecen a estos profesores? ¿Se incluirá una formación pedagógica, además de la académica? ${ }^{33}$. Asimismo, el para qué de la obtención de grados no se deja claro.

Si la propuesta para lograr la calidad educativa es por la vía de la evaluación y de la acreditación: «desempeño y productividad", es obvio que la competitividad institucional no permitirá avanzar a un sistema abierto y flexible.

32 vid., PNE., p.183.

33 vid., GHILARDI, Franco., Crisis y Perspectiva de la Profesión Docente., Gedisa., España., 1993., 159 p. 
Como parte de la conquista en la calidad está el elevar la eficiencia terminal en toda la educación superior. Lo importante no es egresar, sino ¿ cómo egresarán? No está explicado en el PNE. Desde 2001 debía operar el "Programa Integral de Fortalecimiento de las IES", y hasta la fecha (abril 2003) no hay lineamientos ni criterios para ello.

\section{INTEGRACIÓN, COORDINACIÓN Y GESTIÓN DEL SISTEMA DE EDUCACIÓN SUPERIOR}

En el proceso de gestión, lo apremiante a resolver son las dificultades del Bachillerato (en cualquiera de sus modalidades), cómo repercuten en el funcionamiento de la educación superior.

En la gestión del sistema de educación superior, no puede entenderse que el creador de un modelo pedagógico — que multiplica posibilidades de enseñanza y de aprendizaje en miles de profesores y estudiantes- gane menos que quien lo comercializa. Es necesario el apoyo a los investigadores. El año de 2002 inició con la protesta de los miembros del SNI: CONACYT ya declaró que no hay aumento. No se conoce el impacto de las becas, ni cuántas becas crédito han sido otorgadas. Se había propuesto para el 2002 el Sistema Nacional de Evaluación y Acreditación, y el PNE lo propone como meta para el 2006. ¿Dónde queda la proclama de la buena gestión? y por igual, con respecto al sistema abierto $^{34}$, se tendrá que esperar hasta el 2006.

Estudiar las metas del PNE referente al enfoque educativo flexible centrado en el aprendizaje, debía funcionar desde 2001; hasta la fecha no hay ningún impulso sobre ello y en el 2004 se pretende evaluarlo.

En cuanto a planeación, coordinación y gestión con los Estados, aún no se ha articulado. Su prioridad no es la flexibilidad y coordinación de la apertura, sino la formalización de sus funciones ${ }^{35}$.

\footnotetext{
${ }^{34}$ SEP., PNE., p.215.

35 SEP., PNE., p. 211.
} 


\section{PROPUESTAS PARA EVITAR LA INSTRUMENTALI- ZACIÓN DE LA EDUCACIÓN SUPERIOR}

- Edgar Morin propone reformar el pensamiento: pensar globalmente para actuar localmente ${ }^{36}$. México debe pensar así, basándose en las tendencias de los organismos pedagógicos internacionales —que exigen cambios ante un mundo global - para que, localmente, se realicen los cambios (dinámica) educativos, respetando lo permanente (esencia) y no quedarse en ajustes, en modificaciones con respuestas sólo parciales y sin visión del largo plazo. La realidad de la educación superior se reproduce año por año, sexenio tras sexenio y los cambios son lentos y sin rumbo.

- Responder a los procesos de "adaptación" y no de simple "adopción", desarrollando una capacidad de autocrítica de SEP, ANUIES, CONACYT, en lugar de centrarse en el deseo de crear el futuro con un escenario optimista y careciendo de alternativas reales que orienten las líneas estratégicas y el alcance de las metas que sugieren.

- Lograr el don de la ubicuidad en los grandes problemas de la Educación Superior que no están explicitados en los documentos estudiados de SEP y ANUIES:

- Falta de profesionalización de la docencia universitaria. Urge una formación pedagógica para los profesionales, en base a posgrados especializados.

- Impulsar la eficiencia terminal: titulación (posgrado) y graduación (posgrado).

- Vincular los planes y programas de estudio a las necesidades reales del país.

- Desarrollar una cultura de evaluación, como oportunidad de mejora y perfeccionamiento.

- Promover una cultura del aprendizaje, basándose en una educación permanente.

${ }^{36}$ UNESCO., Les clés du XXIe Siecle., p.271. 
- Revalorar la educación técnica ${ }^{37}$.

- México ha llegado tarde a los posgrados; antes eran excepción las personas que los poseían, hoy es necesario incrementarlos creando auténticas Universidades de Posgrado.

- Constituir a la investigación de frontera en el eje articulado de la docencia y de la extensión universitaria.

- Promover que las IES tengan financiamientos alternativos, sobre la base de una educación permanente o continua.

- Es innegable que urge un proyecto de educación superior de orientación humanista.

- En la democracia moderna, la Universidad es indispensable para formar el espíritu público sin el cual la nación no tiene sentido.

- La Universidad es una institución que tiene como su primer deber la búsqueda de la verdad.

- La vocación de la Universidad es inseparable de la idea de trascendencia del mundo del espíritu.

- Como toda institución, la Universidad necesita de un claro concepto de ella misma. Si carece de él, nadie puede ayudarla; la plena economía, la organización y gestión más eficiente no sirve de nada si la Universidad no sabe a qué está destinada. Sólo cuando se reconozca en su misión, sabrá qué le falta atender en los estudiantes, qué señalar a sus profesores, cuáles son los criterios de investigación y de enseñanza (vinculándola para una investigación para la docencia) y sabrá, ante todo, explicar en qué reside la diferencia entre una Universidad y un establecimiento de enseñanza superior.

- La auténtica Universidad sabe que cada uno de sus profesores y de sus estudiantes corresponde a un proyecto personal de vida.

\footnotetext{
${ }^{37}$ En el curso escolar 2000-2001 la educación superior tuvo como matrícula a 2 millones 197,702 estudiantes, el 81\% en Licenciatura de Universidades Públicas, en general $68 \%$ de la matrícula total. El sector privado en los últimos 20 años creció de $15 \%$ al 31.4\%. Por lo tanto, más del $70 \%$ prefiere carreras de corte liberal tradicional con egreso sólo de Licenciatura.
} 
- Contra la instrumentalización de la Universidad, nosotros llamamos a una refundación ética de la misma y a una rehabilitación de su código deontológico. La cuestión crucial debe ser: refundar sobre bases éticas, el conocimiento científico de manera más sólida.

- La revalorización de la investigación de frontera, nos parece una prioridad absoluta, si se quiere que la Universidad continúe siendo un lugar de vinculación de enseñanza e investigación.

- La Universidad del siglo XXI necesita resistirse al burocratismo, al reformismo improvisado, que refute el "clientelismo", y a la demagogia de los discursos políticos.

- Los universitarios deben rescatar el espíritu de la Universidad, retomando en sus manos su destino, haciendo extraordinario lo ordinario.

- La Universidad no debe cubrir sólo el ámbito profesional y científico, sino todos los ámbitos con carácter de integridad; debe ofrecer una nueva Paideia que reconstruya el Ágora; enfatizando la formación de personas humanas y no sólo de técnicos.

- La ciencia sirve a la persona. La educación sirve a la persona para su mejora y su perfeccionamiento. Por ende, sólo se "instrumentaliza" la educación en el servicio a la persona. 


\section{BIBLIOGRAFÍA}

ALTAREJOS, F., et.al.,

Lo permanente y lo cambiante en la educación.

EUNSA., España., 1991., 149 p.

ASOCIACIÓN NACIONAL DE UNIVERSIDADES E INSTITUCIONES DE EDUCACIÓN SUPERIOR (ANUIES)

La Educación Superior hacia el Siglo XXI- Lineas estratégicas de desarrollo.

Documento de trabajo para la XXX Asamblea General. 12 y 13 de noviembre de 1999., 266 p.

COLOM, J. Antoni y DOMÍNGUEZ, Emilia

Introducción a la Política de la Educación.

Ed. Ariel Educación., España., 1997., 299 p.

FOX, Vicente

Discurso de presentación del Programa Nacional de Educación 2001-2006. 28 de septiembre de 2001.

GHILARDI, Franco

Crisis y perspectiva de la profesión docente.

Gedisa., España., 1993., 159 p.

MORIN, Edgar

Les sept savoirs nécessaires á léducation du futur.

Sevil., Paris., 1999., 130 p.

NEEF, Dale

The Knowledge Economy.

Butterworth-Heinemann., Boston., 1998.

ORGANIZACIÓN PARA LA COOPERACIÓN DEL DESARROLLO ECONÓMICO (OCDE)

Apprendre a tout áge, réunion du Comité deléducation au niveau. Ministériel [1996]. 
PICOS MORENO, Arturo

"¿Es posible transformar lo humano?" en ISTMO.

Año 44., No. 259., Marzo-abril, 2002., 28-31 p.

PRAWDA, Juan y FLORES, Gustavo

México Educativo Revisitado.

Océano., México., 2001., 317 p.

RAYNAL, Francoise., et.al.

Pédagogie : dictionnaire des concepts clés.

ESF., Paris., 1997., 405 p.

\section{SANTILLANA}

Diccionario de las Ciencias de la Educación.

Santillana., España., 1945., 1431 p.

SECRETARÍA DE EDUCACIÓN PÚBLICA (SEP)

Bases para el Programa 2001-2006 del Sector Educativo.

Lunes 27 noviembre de 2000 (Reforma)

SECRETARÍA DE EDUCACIÓN PÚBLICA (SEP)

Programa Nacional de Educación 2001-2006.

SEP., México., Septiembre 2001., 269 p.

TORRES, Juan Ángel

- Universidad Virtual.

Ed. Delfos., México., 2001., 224 p.

- La educación superior en el siglo XXI: Visión y acción.

UNESCO., Paris., 1998., 137 p.

- Les clés du XXIe siècle.

UNESCO., Paris., 2000., 515 p.

- El derecho a la educación.

Santillana., España., 2000., 183 p.

- Marco de acción de Dakar.

UNESCO., Paris., 2000., 78 p. 